\title{
Jet Flow Characteristics of 3-Slot Nozzle in Gas Wiping Process at Continuous Galvanizing Line
}

\author{
Gentaro TAKEDA, ${ }^{*}$ Hideyuki TAKAHASHI and Kazuhisa KABEYA \\ Rolling \& Process Research Dept., Steel Research Laboratory, JFE Steel Corporation, 1 Kokan-cho, Fukuyama, Hiroshima, 721 - \\ 8510 Japan
}

(Received on December 7, 2016; accepted on February 23, 2017; originally published in Tetsu-to-

Hagané, Vol. 102, 2016, No. 10, pp. 576-582)

\begin{abstract}
This paper presents a study of the gas wiping process, which is used in coating processes to control the final coating weight applied on a substrate. The wiping process is one of the factors which limit the maximum line speed of continuous galvanizing lines (CGL). In recent years, a multi-slot type wiping nozzle was proposed to improve gas wiping performance, and was investigated by numerical simulation. Here, the impinging jet characteristics of a 3-slot nozzle are investigated by experimental and numerical analysis, focusing on the mixing process of the jets and the distribution of the impinging pressure of the mixed jet. The gas wiping capability was tested in model wiping experiments with a molten paraffin coating. The results confirmed that the impinging pressure distribution of a 3-slot nozzle with a relatively low auxiliary jet velocity is sharper than that of a single-slot nozzle, and this makes it possible to reduce the coating weight. Conversely, when the jet velocity of the auxiliary slots approaches that of the main slot jet, the width of the impinging pressure distribution spreads and the coating weight increases. The turbulent kinetic energy of the flow field changes depending on the velocity of the auxiliary jets, and the energy distribution decides the width of the mixed jet flow.
\end{abstract}

KEY WORDS: hot-dip coating; continuous galvanizing line (CGL); gas wiping; air knife; coating weight; multiple-slot jets.

\section{Introduction}

Gas wiping is used for coating weight control in Continuous Galvanizing Lines (CGL) which produce coated steel sheets for automobiles, building materials and electric appliances. Because the impinging gas jet wipes the excess molten zinc from the steel strip, gas wiping provides a noncontact method for controlling the strip coating weight. Gas wiping has advantages such as uniformity in the width direction, the ability to produce a thin plating and economy. Usually, a single-slot gas nozzle (hereinafter, 1-slot nozzle) is installed above the molten zinc pot. Numerous studies have examined the characteristics of the two-dimensional jet flow of 1-slot nozzles and mathematical analysis of the gas wiping mechanism by both experiments and computational fluid dynamics. $^{1-4)}$

From those results, control of the gas jet pressure in gas wiping should make it possible to increase the CGL line speed in order to realize higher productivity. However, the maximum CGL line speed remains at around $3.0 \mathrm{~m} / \mathrm{s}$, whereas the line speed of continuous annealing lines (CAL), which do not include a zinc coating process, exceeds 4.0 $\mathrm{m} / \mathrm{s}$. The main obstacles to higher CGL line speeds are the need for a thin plating capability which enables thickness

* Corresponding author: E-mail: g-takeda@jfe-steel.co.jp DOI: http://dx.doi.org/10.2355/isijinternational.ISIJINT-2016-715 control to the level of several $\mu \mathrm{m}^{5)}$ and the problem of the surface defect called splashing, which occurs during gas wiping. ${ }^{6)}$

In recent years, multi-slot gas nozzles have been investigated with the aim of overcoming these problems. Tamadonfer et al. ${ }^{7}$ and Dubois ${ }^{8)}$ carried out numerical simulations on the jet characteristics and wiping performance of a 3-slot nozzle with two auxiliary slots arranged on the two sides of the main slot, which was perpendicular to the steel sheet. Although the simulation results indicated that the maximum impinging pressure of the 3-slot nozzle is higher than that of the 1-slot nozzle, the coating weight after wiping with the 3-slot nozzle was thicker than that after the 1-slot nozzle. Takeda et al. ${ }^{9)}$ first investigated the jet flow characteristics and wiping performance of 3-slot nozzles in an experimental analysis with a paraffin wiping apparatus. The results showed that the jet formation after mixing of the three jets changes depending on the shape of the nozzle tips, and as a result, wiping performance might improve or deteriorate, depending on the nozzle design. However, the influence on wiping performance and changes in the formation of the three jets depending on the velocity of the auxiliary slot jets were not clarified.

The main objective of the present study is to clarify the mechanism of jet mixing with a 3-slot nozzle and the effect of jet mixing characteristics on coating weight. The impinging pressure distribution was measured under several 
pressure conditions using the 3-slot nozzle used in the previous studies, ${ }^{9-11)}$ and wiping performance was examined by using the same model wiping apparatus as in the previous studies. In addition, the process of jet formation with the 3-slot nozzle was analyzed in detail by computational fluid dynamics (CFD).

\section{Mathematical Model of Coating Thickness}

Ellen and $\mathrm{Tu}^{3)}$ suggested a mathematical model for the coating thickness as follows. As shown in Fig. 1, the impinging pressure of the gas wiping jet on a steel strip causes a pressure gradient, and this pressure gradient and the shear stress of the wall jet that flows along the steel strip surface act on the molten zinc layer adhering to the strip after the strip passes through the zinc pot. The liquid film thickness is predicted by this gas wiping mechanism under the following basic assumptions:

(1) The fluid flow in the liquid coating film flow can be described by a steady state, two-dimensional equation of incompressible, constant viscosity. The flow that is perpendicular to the strip is small enough for the flow that is parallel to the strip.

(2) Surface tension, oxidation effects, strip surface roughness and interfacial alloy formation can be neglected.

(3) There is no slip between the fluid coating and the strip at the strip-fluid interface.

The simplified Navier-Stokes equation for the liquid film is written as:

$$
\mu_{L} \frac{d^{2} u_{L}}{d y^{2}}=\rho_{L} g+\frac{d P}{d x}
$$

Based on the mass conservation law, the volumetric flow of the coating liquid per unit width of strip $q$ is written as:

$$
q=\int_{y=0}^{y=t} \quad u_{L}(x, y) d y
$$

The boundary conditions for solving the above equations are written as:

$$
\begin{gathered}
u_{L}=V \quad \text { at } \quad y=0 \ldots \\
\mu_{L} \frac{d u_{L}}{d y}=\tau \quad \text { at } \quad y=t
\end{gathered}
$$

Integrating Eqs. (1) and (2) and applying the boundary conditions (3) and (4) results in the following solution:

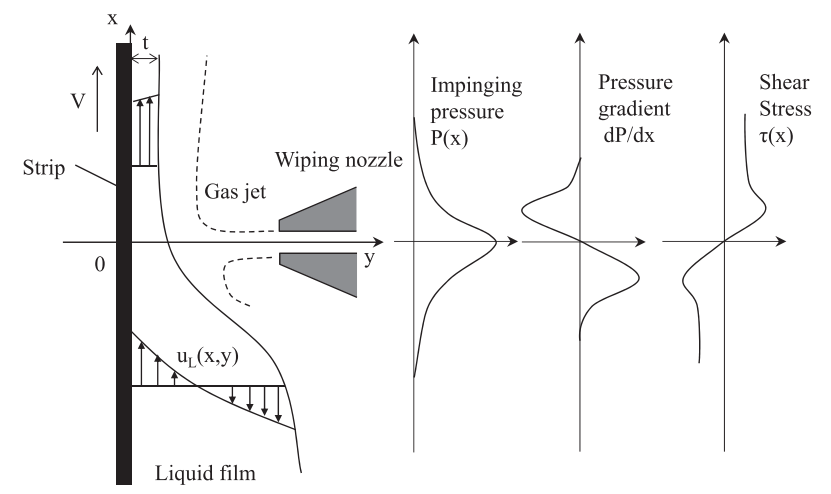

Fig. 1. Analytical model of gas wiping.

$$
T=\frac{S+\sqrt{S^{2}+4 G}}{2 G}
$$

where,

$$
\begin{array}{r}
T=t \sqrt{\rho_{L} g / \mu_{L} V} \\
S=\tau / \sqrt{\rho_{L} \mu_{L} V g} \ldots . \\
G=1+(d p / d x) / \rho_{L} g .
\end{array}
$$

$T, S$ and $G$ are the non-dimensional numbers related to gas wiping. $T$ is the non-dimensional film thickness, $S$ is the non-dimensional wall shear stress, $G$ is the non-dimensional pressure gradient, $\mu_{L}$ is the viscosity of the coating liquid, $u_{L}$ is the liquid film velocity in $x$ direction, $\rho_{L}$ is the density of the coating liquid, $g$ is the acceleration of gravity, $P$ is the impinging pressure, $t$ is the local film thickness and $V$ is the strip travel velocity. As described by Eq. (5), the pressure gradient (forced by the wiping jet) $d p / d x$ and wall shear stress $\tau$ decide the coating film thickness. In the case of a 1 -slot nozzle, the combination of the slot nozzle gap, the jet pressure and the distance between the strip and the nozzle tip uniquely determine $d p / d x$ and $\tau{ }^{1)}$ On the other hand, there are few studies on the jet characteristics of multi-slot nozzles such as 3-slot nozzles. As similar phenomena, studies on the mixing/diffusion behavior of a coaxial circular jet $^{12)}$ near a multi-slot nozzle preceded research on multislot nozzles.

\section{Experimental Procedure and Numerical Analysis}

\subsection{3-slot Nozzle}

In comparison with 1-slot nozzles, the jet characteristics of 3-slot nozzles are affected by a number of parameters, such as the nozzle geometry and gas jet conditions. A previous study ${ }^{9)}$ by some of the same authors indicated that the distance between the main slot and the auxiliary slots has a remarkable influence on the formation of the jets after the three jets merge. In particular, wiping performance was improved by shortening the distance between the main slot and the auxiliary slots.

Figure 2 shows a schematic diagram of the tip shape of the 3-slot gas wiping nozzle used in this paper. Based on the previous report, the distance between the main slot and the upper/lower auxiliary slots was set at $0.1 \mathrm{~mm}$. In addition, it is estimated that the jet angle of the auxiliary slot (angle of the main slot and the auxiliary slots) greatly influences jet formation. Since Takahashi et al. ${ }^{13)}$ showed that wiping performance improves as the nozzle outer angle becomes more acute, wiping performance is expected to change due to factors other than the slot jet if the outer angle is changed from the $50^{\circ}$ of the nozzle used in the previous study. There-

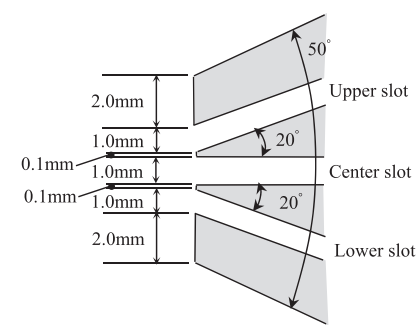

Fig. 2. Tip shape of 3-slot gas wiping nozzle. 
fore, in the present study, one kind of 3-slot nozzle with a nozzle outer angle of $50^{\circ}$ and upper/lower nozzles with a jet angle inclinations of $20^{\circ}$ arranged symmetrically above/ below the center slot was tested. A photograph of this nozzle is shown in Fig. 3.

Compressed air pressurized by a compressor was used for the wiping gas. The compressed air was adjusted to the predetermined pressure by a reducing valve, and was then supplied to the three slots by a distribution header. A ball valve was attached to each pipe after the distribution header, making it possible to adjust the nozzle header pressure of the center slot and the auxiliary slots as desired. A uniform air flow was obtained by passing the air through a rectification aperture installed in the nozzle, after which the air was discharged from the nozzle via the slots. Here, gaps D of the center, upper and lower slots were all $1.0 \mathrm{~mm}$, and the slot width was $250 \mathrm{~mm}$.

\subsection{Experimental Gas Wiping Simulator}

Wiping performance was investigated by using the previously-developed gas wiping simulator. ${ }^{9-11)}$ The arrangement of the experimental apparatus is shown in Fig. 4. In this apparatus, the strip in coil form is paid off from the pay-off reel and immersed continually in the coating liquid bath. The coating weight of the liquid is controlled by mutuallyopposed wiping nozzles arranged above the coating bath, and the strip is then coiled by the coiler. Wiping conditions such as the nozzle jet angle, wiping gas pressure and distance between the strip and nozzle can be set optionally. In this experiment, the tilt angles of the two wiping nozzles were fixed at $0.0^{\circ}$ (i.e., horizontal).

Paraffin having a melting point of approximately $60^{\circ} \mathrm{C}$ was used as the coating liquid, and the bath temperature during the experiment was $90^{\circ} \mathrm{C}$, that is, approximately $30^{\circ} \mathrm{C}$ higher than the melting point. The liquid paraffin cooled about $10^{\circ} \mathrm{C}$ while passing the wiping nozzles but did not solidify before the strip arrived at the top roll, which was

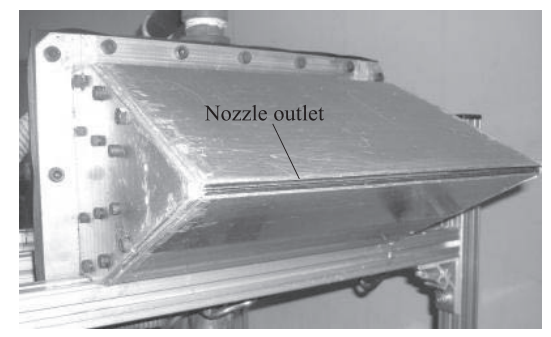

Fig. 3. Photograph of 3-slot wiping nozzle.

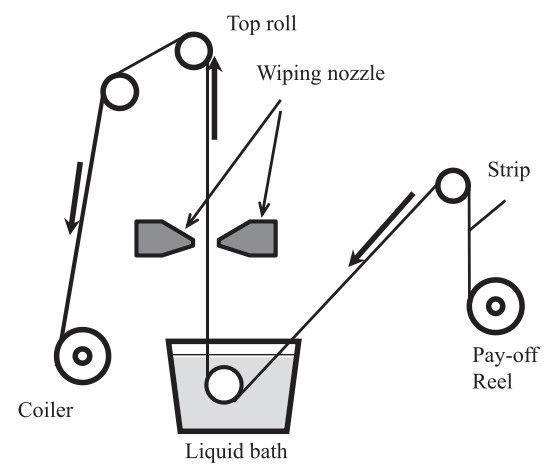

Fig. 4. Experimental gas wiping apparatus. located $1.5 \mathrm{~m}$ above the wiping nozzles. Table 1 shows a comparison of the physical properties, gas wiping conditions and non-dimensional numbers $T, G$ and $S$ shown in Chapter 2 of the molten zinc used in the actual CGL and the paraffin used in this experiment. Because the non-dimensional numbers $T, S$ and $G$ of the simulator were within the ranges of those of the CGL, and the coating thickness obtained with the simulator was in good agreement with the value predicted from the mathematical model mentioned above, it is thought that hydrodynamic similarity is established between the CGL and the simulator in the range of $0.03 \leqq T \leqq 0.04$, $0.6 \leqq S \leqq 1.0$ and $230 \leqq G \leqq 460$. When zinc is coated on a steel strip, an alloying reaction may occur. However, no such reaction occurs at the interface of the paraffin and the metal. This means that verification purely in terms of gas wiping theory is possible.

Coating weight can be measured by various methods, such as measurement of the coating film cross section, observation of samples, comparison of the coating weight before/after delamination (hereinafter, weight measurement method) and measurement with an eddy current-type contact film thickness meter. In this experiment, the weight measurement method was adopted in order to quantify the mean coating weight of a relatively large area precisely.

\subsection{Measurement of Impinging Pressure}

The method of measuring the impinging pressure of the two-dimensional jet will be described referring to Fig. 5. The plate on which the jet impinges (hereinafter, impinging plate) is a stainless steel plate with the thickness of $1 \mathrm{~mm}$, width of $300 \mathrm{~mm}$ and height of $300 \mathrm{~mm}$. A measurement hole of $\Phi 0.5 \mathrm{~mm}$ was drilled in the plate center, and a pressure sensor was attached to the back side of the plate. The distance between the nozzle tip and the impinging plate was $10 \mathrm{~mm}$, which is the same as that in the wiping simulator experiment. The impinging pressure was measured by attaching the impinging plate to a XY stage and moving the plate in the vertical direction. The repetitive positioning precision of this $\mathrm{XY}$ stage is $\pm 10 \mu \mathrm{m}$. The measurement time

Table 1. Physical properties of coating materials, conditions of coating process and dimensionless numbers.

\begin{tabular}{lcc}
\hline & CGL & Wiping simulator \\
\hline Coating material & Zinc & Paraffin ${ }^{* 1}(\mathrm{HNP}-5)$ \\
Operation temperature $\left[{ }^{\circ} \mathrm{C}\right]$ & 460 & 90 \\
Coating density $\left[\mathrm{kg} / \mathrm{m}^{3}\right]$ & 6623 & 759 \\
Coating viscosity $[\mathrm{Pa} \cdot \mathrm{s}]$ & 0.00294 & 0.0071 \\
Coating surface tension $[\mathrm{N} / \mathrm{m}]$ & 0.81 & $0.0026^{* 2}$ \\
Nozzle - strip distance $[\mathrm{mm}]$ & $5-10$ & 10 \\
Transfer speed $[\mathrm{m} / \mathrm{s}]$ & $1.0-2.5$ & 0.67 \\
Plenum pressure $[\mathrm{kPa}]$ & $10-70$ & 1.5 (center slot) \\
$T$ & $0.024-0.074$ & $0.03-0.04$ \\
$S$ & $0.5-3.0$ & $0.6-1.0$ \\
$G$ & $50-500$ & $230-460$ \\
\hline
\end{tabular}

*1 NIPPON SEIRO CO. HNP-5

*2 Maker's publication 
per point was $5 \mathrm{~s}$, and the impinging pressure was evaluated by the mean value.

\subsection{Numerical Simulation of Wiping Gas Jet}

The formation of the jet from the nozzle slot to the strip was investigated by two-dimensional steady analysis using the commercial code Fluent 14.5. Realizable k-Ewas adopted as the turbulent model because it is considered more suitable for analysis of the two-dimensional jet than the standard k- $\varepsilon$ model. ${ }^{14)}$ Enhanced wall treatment, which combines the standard wall function model with the near wall model, was adopted for treatment of the near wall. ${ }^{15)}$ The calculation mesh is shown in Fig. 6. The mesh number was approximately 80,000 .

\section{Experimental Results and Discussion}

\subsection{Influence of Auxiliary Slot Jets on Wiping Perfor- mance}

The paraffin wiping experiment was carried out under the conditions shown in Table 1. The header pressure of the center slot was fixed at $1.5 \mathrm{kPa}$, and the upper/lower slot jets were injected individually or simultaneously at pressures from 0.2 to $1.0 \mathrm{kPa}$.

Figure 7 shows the experimental results. The results for the upper/lower slot pressures of $0.0 \mathrm{kPa}$ indicate the coating weight in the case of injection only from the center slot. In comparison with the cases of individual injection of the jet from the upper or lower slot, the greatest improvement in wiping performance was achieved in the case of simultaneous jetting from the upper/lower slots in this gas pressure

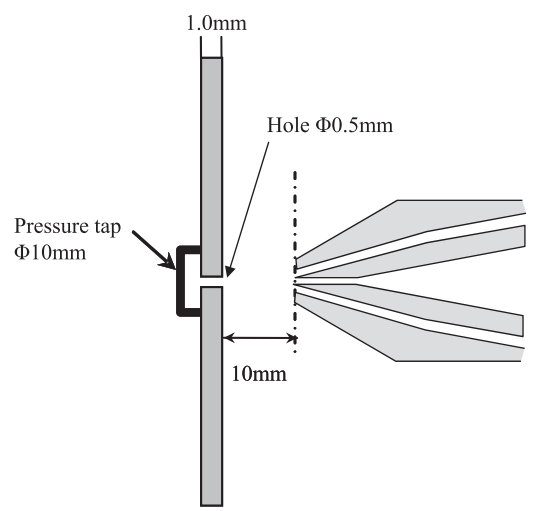

Fig. 5. Schematic diagram of impinging pressure measurement.

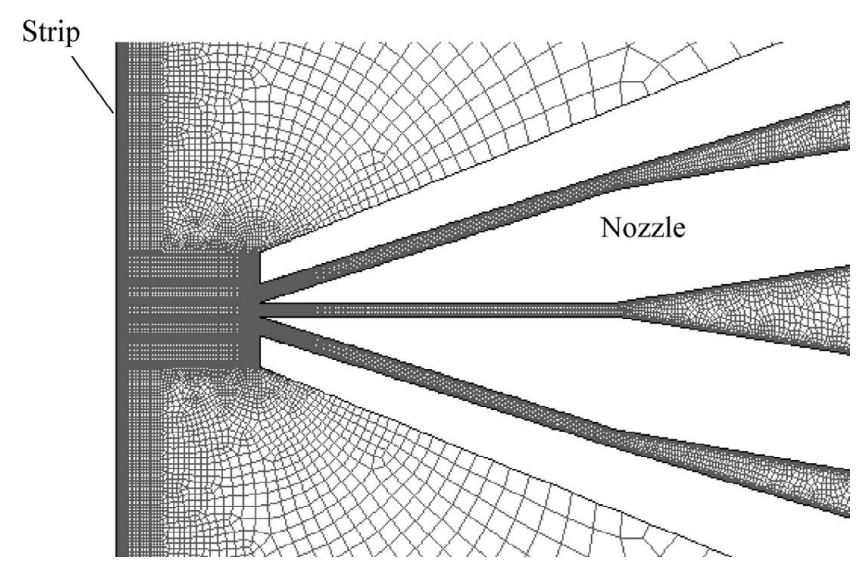

Fig. 6. Example of mesh for CFD analysis. range, and a thin coating film was obtained. Comparing injection of only the upper slot jet with only the lower slot jet, a somewhat thinner paraffin thickness was achieved with the upper slot jet. Focusing the influence of gas pressure, the coating weight was the lowest at the upper or lower slot pressure of $0.2 \mathrm{kPa}$ with all jet patterns, and the coating weight increased when the gas pressure exceeded $0.2 \mathrm{kPa}$. With the conventional 1-slot nozzle, the coating weight decreases as the wiping gas pressure increases, as predicted from the mathematical model of gas wiping presented in Chapter 2. However, with the 3-slot nozzle, these results showed that increasing the jet pressure does not necessarily lead to an improvement in wiping performance due to variations in the formation of the center jet flow caused by the upper/lower slot jets.

Figure 8 shows the impinging pressure distributions measured by the method in Fig. 5. Here, the $\mathrm{x}$ direction position on the horizontal axis is translated to a non-dimensional position by the slot gap D. As the slope of the impinging pressure distribution becomes steeper, the non-dimensional number $G$ in the gas wiping equation in Chapter 2 increases, which means that wiping performance improves. Therefore, the jet characteristics at the upper and lower slot pressure of $0.2 \mathrm{kPa}$, which reduced the coating weight in comparison with the 1-slot nozzle, and the jet characteristics at $1.0 \mathrm{kPa}$, which increased the coating weight in comparison with the 1 -slot nozzle, were investigated in detail. Figure 8(a) shows the measured impinging pressure distributions when the center, upper and lower slots are jetted together. In comparison with jetting of only the center slot, when the pressure of the upper/lower slots is $0.2 \mathrm{kPa}$, the maximum pressure increases without an accompanying increase in the width of the impinging pressure distribution, and the impinging pressure distribution is steeper than the distribution with only the center slot. However, at the upper/lower slot pressure of $1.0 \mathrm{kPa}$, a further increase in the maximum impinging pressure is observed, but the width of the impinging pressure distribution also increases, becoming more than double, and the pressure gradient becomes somewhat more moderate. It is assumed that the measured pressure distribution did not display a perfect symmetrical distribution because the tested nozzle did not have a perfect symmetrical shape. Figure 8(b) shows the measured impinging pressure distributions

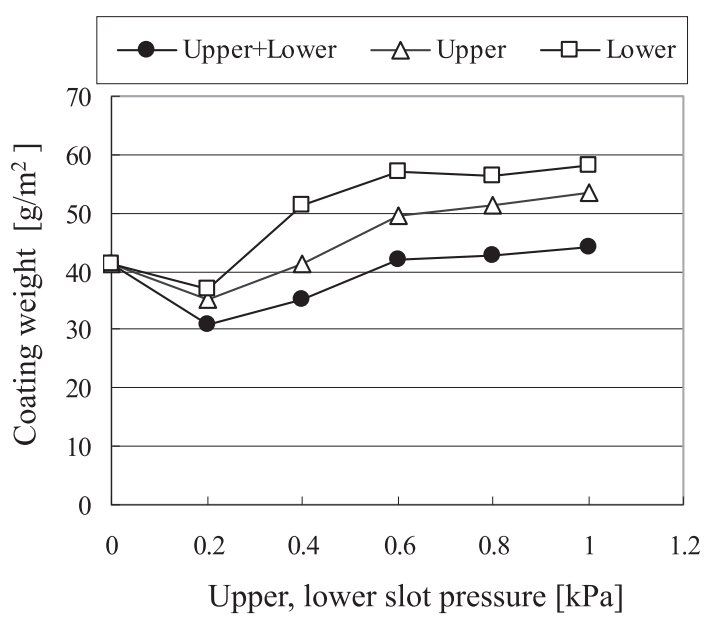

Fig. 7. Coating weight in paraffin wiping experiments with 3-slot wiping nozzle. 

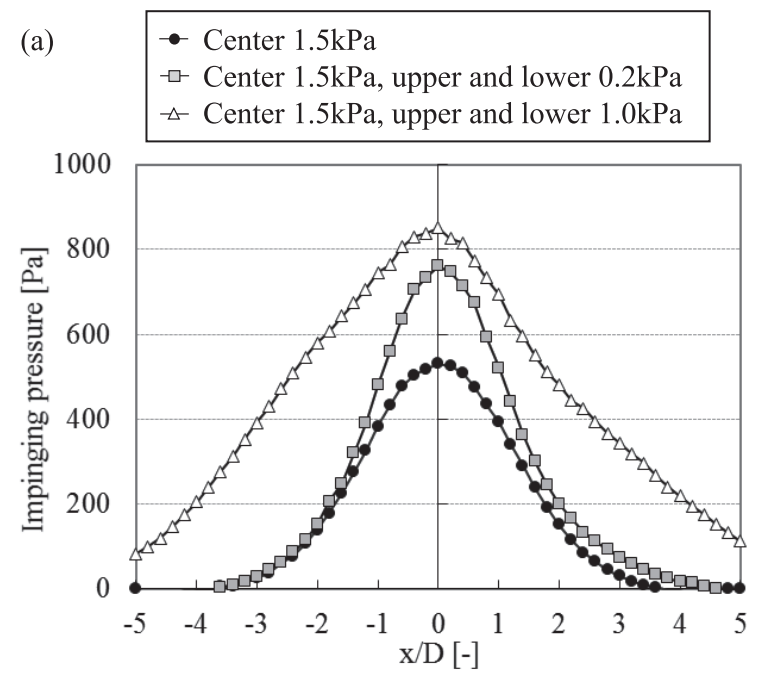
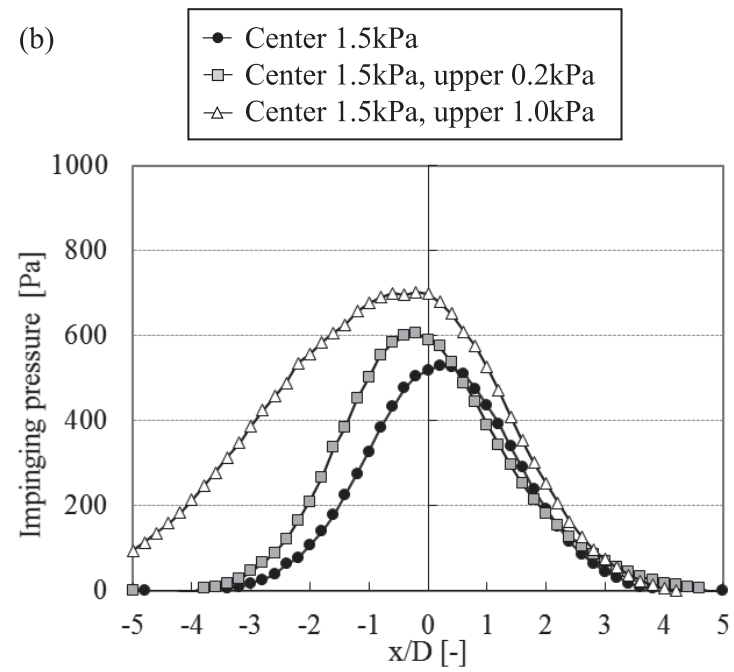

Fig. 8. Measured impingement pressure distribution on strip surface.
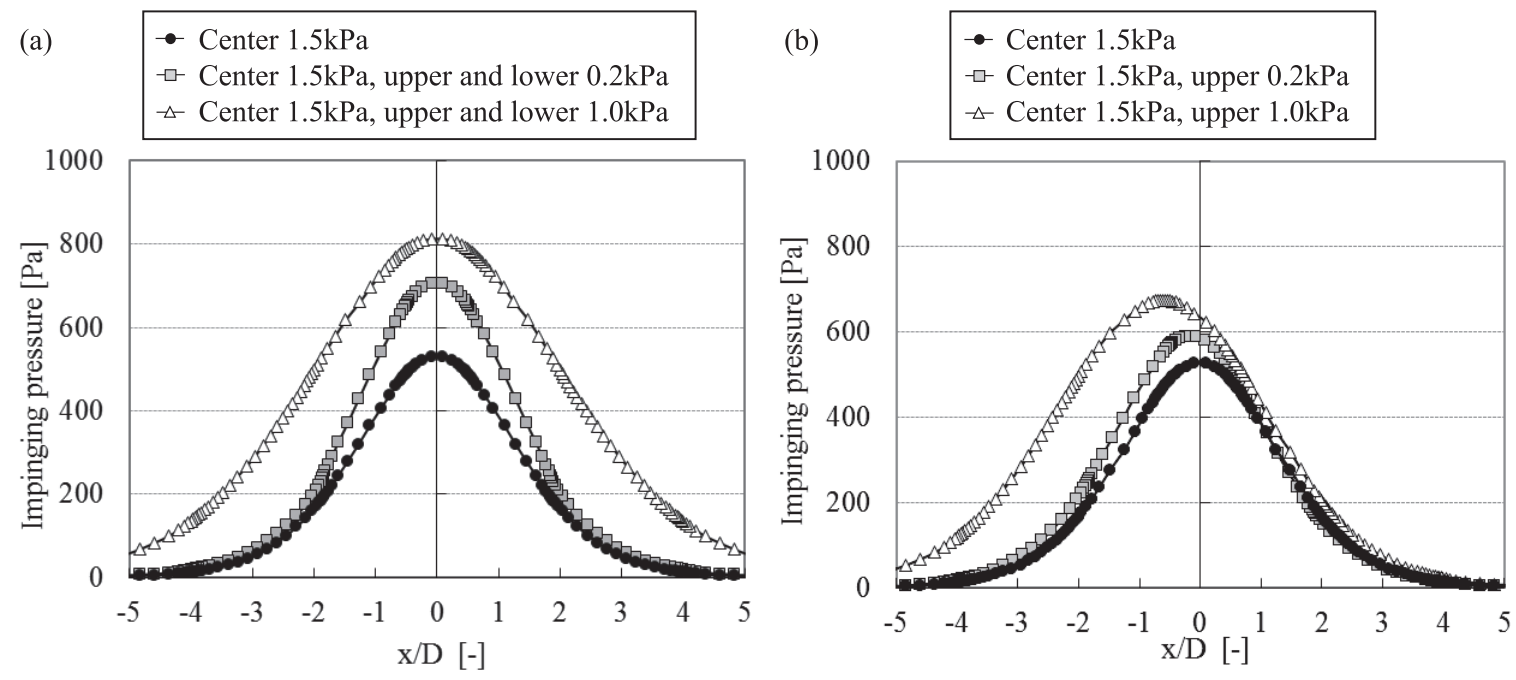

Fig. 9. Calculated impingement pressure distribution on strip surface.

when the center and upper slot were jetted together. Due to the upper slot jet, the pressure distribution becomes asymmetrical, and the $\mathrm{x}$ position of the maximum pressure shifts slightly to the lower side $(\mathrm{x} / \mathrm{D}<0)$ of the center slot position. Furthermore, the jet flow is formed with different pressure gradients on the upper side $(\mathrm{x} / \mathrm{D}>0)$ and the lower side $(\mathrm{x} / \mathrm{D}<0)$. It is estimated that this variation in jet formation will influence wiping performance.

Figure 9 shows the calculated impinging pressure distributions obtained by CFD corresponding to the conditions in Fig. 8. Figure 10 shows a comparison of the pressure half-width $\mathrm{y}_{\mathrm{p} 1 / 2}$ (jet flow width at half of maximum impinging pressure) in the measured results (Fig. 8) and the CFD results (Fig. 9). The half-width in the CFD results is slightly small at the upper/lower slot pressure of $1.0 \mathrm{kPa}$, and the distributions do not correspond completely. However, from the tendency of the changes in the pressure half-width in the CFD analysis, it is judged the CFD can approximately reproduce the experimental results. Therefore, in the next section, the jet flow mixing behavior under the various conditions will be investigated by using CFD.

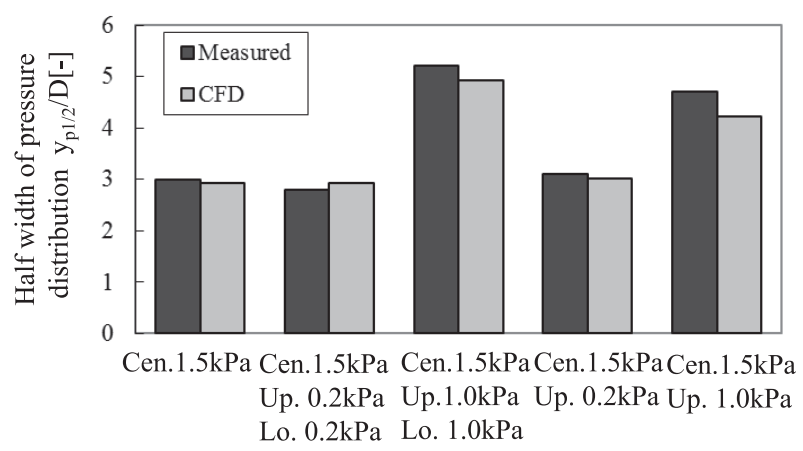

Fig. 10. Comparison of half width of pressure distribution.

\subsection{Jet Flow Formation of 3-slot Nozzle}

As an example of the formation of the jet flow by mixing of the flows from the three slots from the nozzle tip to the strip, the velocity contours calculated by CFD are shown in Fig. 11(a) for the center slot pressure of $1.5 \mathrm{kPa}$, in (b) for the center slot pressure of $1.5 \mathrm{kPa}$ and upper/lower slot pressure of $0.2 \mathrm{kPa}$ and in (c) for the center slot pressure of $1.5 \mathrm{kPa}$ and upper/lower slot pressures of $1.0 \mathrm{kPa}$. In this simulation, the gas jet velocity is $42 \mathrm{~m} / \mathrm{s}$ at $1.5 \mathrm{kPa}, 15 \mathrm{~m} / \mathrm{s}$ at $0.2 \mathrm{kPa}(35.7 \%$ of center slot velocity) and $32 \mathrm{~m} / \mathrm{s}$ at 1.0 
$\mathrm{kPa}(76.2 \%$ of center slot velocity). In comparison with (a), the decrement of the center slot jet weakens in (b) and (c), and the high jet velocity is kept more to the rear. Thus, in the region near the nozzle, it is possible to compare the jet flow mixing behavior by using the velocity distribution. However, because the dynamic pressure of the jet flow is converted to static pressure on the strip in the wall jet region, and the jet directions and half-width of the wall jet also change, a simple comparison of mixture behavior by using only the velocity distribution is difficult. Therefore, in this paper, the jet mixture process was investigated by the total pressure distribution, which was defined as the sum of the dynamic pressure and static pressure in the CFD analysis. The total pressure transition from the nozzle tip to the near-strip region $(\mathrm{y} / \mathrm{D}=0-8$, nozzle tip is $\mathrm{y}=0)$ is shown in Fig. 12. This figure shows the calculated result for (a) only center slot with pressure of $1.5 \mathrm{kPa}$ (1 jet), (b) and (c); center slot + upper/lower slots ( 3 jets) and (d) and (e); center slot + upper slot (2 jets).

In (b), where the upper/lower slot pressure is $0.2 \mathrm{kPa}$, dynamic pressure peaks due to the upper and lower slot jets appear can be seen after jetting $(\mathrm{y} / \mathrm{D}=0)$, but these peaks disappear quickly at $y / D=2$ due to merging of the three jets, and the jet take a shape approaching that of single jet. Because the velocity difference between the center jet and the ambient gas is reduced by the slow jets from the upper/ lower sides, the decrement of the center jet is suppressed and the impinging pressure near the strip increases. Wiping performance is improved by this kind of jet formation. In (c), where the upper/lower slot pressure is $1 \mathrm{kPa}$, the peaks caused by the upper/lower jets remain at $y / D=4$. After this point, complete mixing occurs and jet diffusion progresses. Under this condition, the pressure distribution is gentler than that of the 1-slot jet.

In (d), which is a 2-slot arrangement with the upper slot pressure of $0.2 \mathrm{kPa}$, jet formation is the same as in (b) on the upper side $(x / D>0)$, and is the same as (a) on the lower side $(\mathrm{x} / \mathrm{D}<0)$. In this case, the peak of total pressure does not increase so effectively because suppression of the diffusion of the center jet is insufficient with only one auxiliary jet. These results indicate that supplying slow auxiliary jets from both sides of a center jet is effective for suppressing diffusion of the main slot jet. In (e), which is the 2-slot arrangement with the upper slot pressure of $1.0 \mathrm{kPa}$, the pressure gradient at $\mathrm{x} / \mathrm{D}<0$ changes to an extremely sharp form due to deviation of the center jet to the lower side. This behavior can be observed until about the $y / D=4$ position, that is, before the two jets mix completely. Although this jet pattern might appear to improve wiping performance, the pressure gradient moderates quickly after the two jets mix, and wiping performance deteriorates at $\mathrm{y} / \mathrm{D}=10$.

In order to estimate the diffusions of the two-dimensional

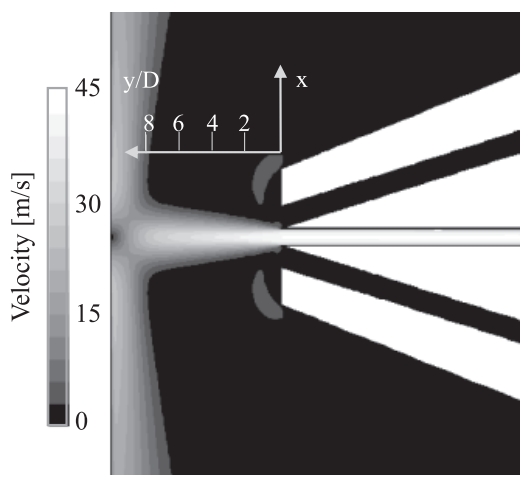

(a)Center $1.5 \mathrm{kPa}$

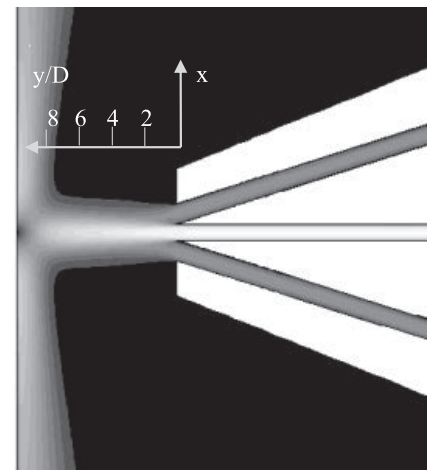

(b)Center $1.5 \mathrm{kPa}$, upper and lower $0.2 \mathrm{kPa}$

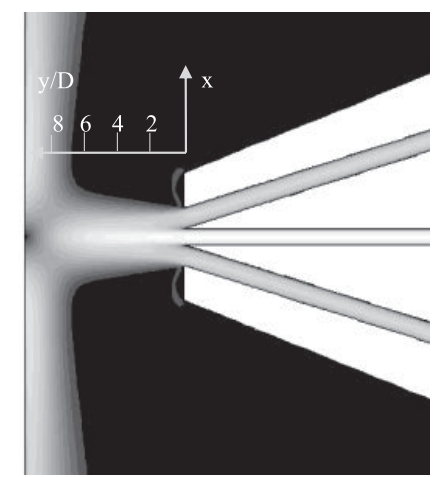

(c)Center $1.5 \mathrm{kPa}$, upper and lower $1.0 \mathrm{kPa}$

Fig. 11. Velocity contours of 1-slot and 3-slot jets.

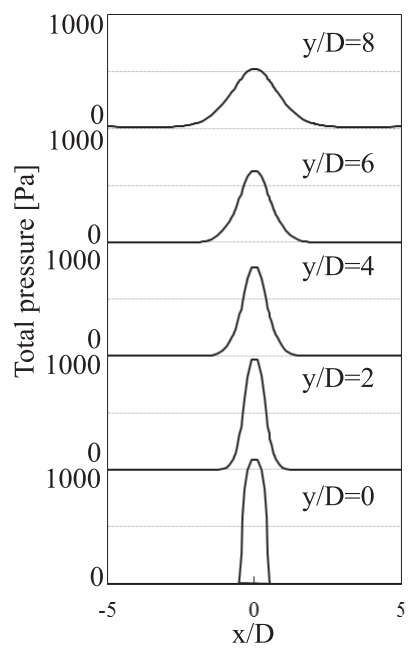

(a) Cen. $1.5 \mathrm{kPa}$

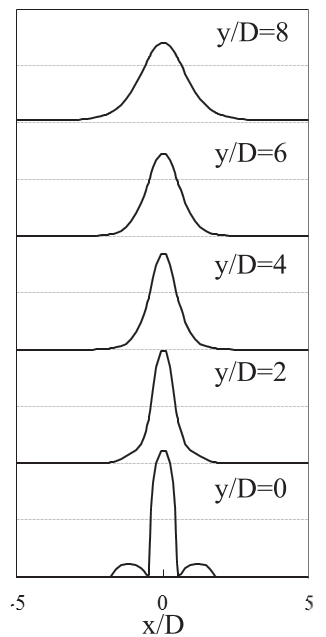

(b) Cen. $1.5 \mathrm{kPa}$,

Up. $0.2 \mathrm{kPa}$, Lo. $0.2 \mathrm{kPa}$

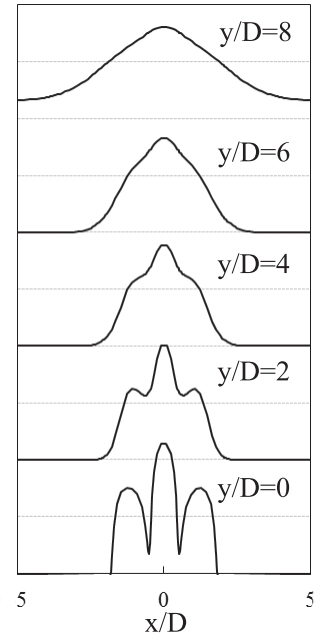

(c) Cen. $1.5 \mathrm{kPa}$

Up.1.0kPa, Lo. $1.0 \mathrm{kPa}$

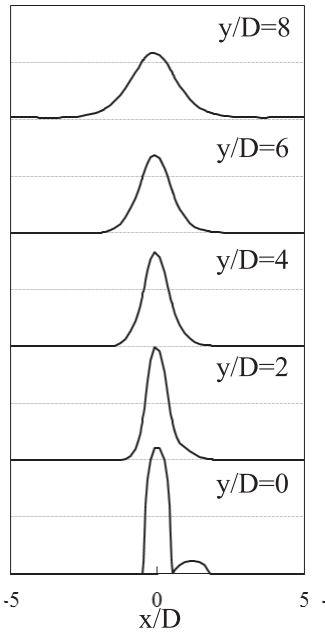

(d) Cen. $1.5 \mathrm{kPa}$, Up. $0.2 \mathrm{kPa}$

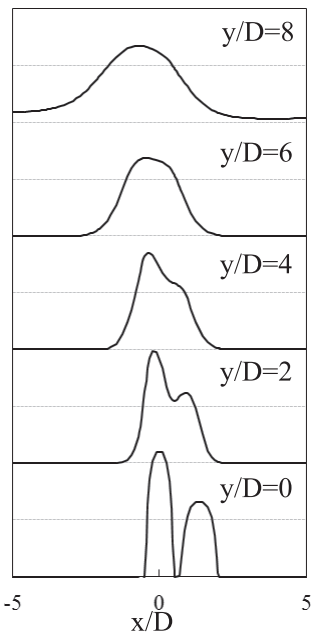

(e) Cen.1.5kPa, Up. $1.0 \mathrm{kPa}$

Fig. 12. Total pressure distributions between nozzle tip and strip with 1-slot, 2-slot and 3-slot jets. 


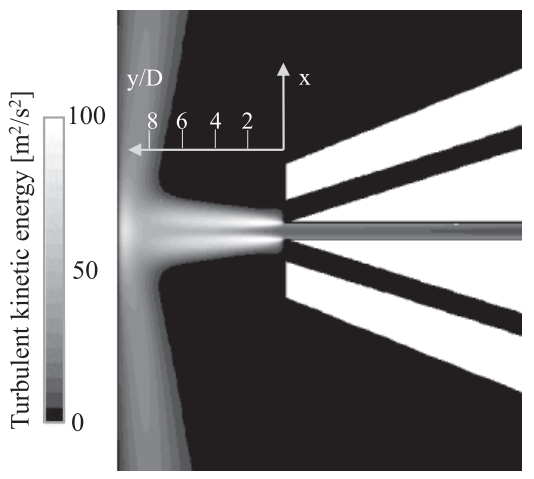

(a)Center $1.5 \mathrm{kPa}$

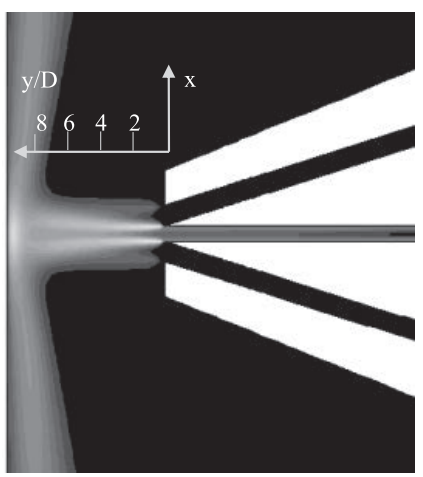

(b)Center $1.5 \mathrm{kPa}$, upper and lower $0.2 \mathrm{kPa}$

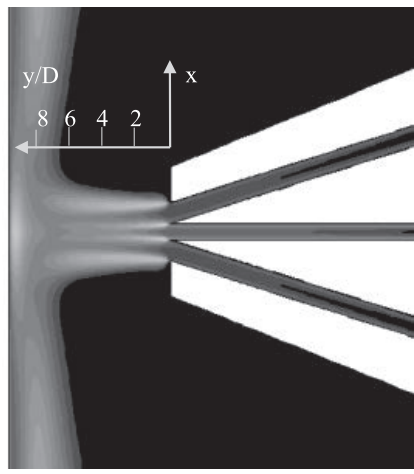

(c)Center $1.5 \mathrm{kPa}$, upper and lower $1.0 \mathrm{kPa}$

Fig. 13. Turbulent kinetic energy contours of 1-slot and 3-slot jets.

jet flow discussed above more directly, the turbulent kinetic energy ( $\mathrm{k}$ of $\mathrm{k}-\varepsilon$ model) calculated by CFD will be compared. Figure 13 represents the turbulent kinetic energy distributions under the same conditions as in Fig. 11. For the center slot jet, under condition (a), i.e., jetting into a static gas, the large velocity difference on the two sides of the jet causes strong turbulent kinetic energy. On the other hand, under condition (b), that is, low-speed jetting with auxiliary jets, the turbulent kinetic energy on the two sides of the center slot jet decreases due to the lower velocity difference with the ambient gas. Moreover, since remarkable turbulent kinetic energy is not generated on the outer sides of the upper/lower slot jets, this energy does not have a large influence on the jet width after mixing. Comparing the integral value of the turbulent kinetic energy in the flow field from the nozzle tip to the strip $(-4 \leqq x / D \leqq 4,0 \leqq y / D \leqq 10)$, assuming the value of (a) is 100 , the value of (b) is reduced to 79.3 , or a reduction of about $20 \%$. However, in the case of (c), which is the case of high-speed auxiliary jets, the width of the mixed jet increases to the equivalent of the width of the three jets due to the increased turbulent kinetic energy at the outside of the auxiliary jets in comparison with that at the sides of the center jet. As a result, the integral value of (c) is 84.7. In other words, the turbulence in the total flow field increases under condition (c) in comparison with (b).

\section{Conclusion}

In order to clarify the function of the auxiliary slot jets in the wiping performance of a 3-slot nozzle, gas wiping experiments were performed with liquid paraffin, the impinging pressure of the wiping gas jet was measured and a CFD analysis were carried out. The results are summarized as follows.

(1) When the center slot pressure was $1.5 \mathrm{kPa}$, wiping performance was improved by upper/lower slot pressures in the range of $0.2-0.4 \mathrm{kPa}$. However, wiping performance gradually deteriorated with further increases in the upper/ lower slot pressure. Simultaneous use of the upper and lower slot jets improved wiping performance.

(2) The results of measurements of the impinging pressure indicated that the pressure gradient is sharp under jet conditions that improve wiping performance (upper/lower slot pressure: $0.2 \mathrm{kPa}$ ), and the pressure gradient is moderate under jet conditions that deteriorate wiping performance (upper/lower slot pressure: $1.0 \mathrm{kPa}$ ).

(3) The CFD analysis revealed that the velocity difference between the center slot jet flow and the ambient gas decreases due to the auxiliary slot jets, and diffusion of the jet flow is suppressed due to a reduction in the turbulent kinetic energy at the two sides of the center slot jet.

(4) If the auxiliary jet velocity is set low, for example, to around $35 \%$ of the center jet velocity, the jet width does not spread after mixing of the 3 jets. However, if the auxiliary slot jet velocity is increased, the outer edge of the auxiliary slot jets becomes the outer edge of the mixed jet, and in effect, the width of the mixed jet spreads. As a result, the impinging pressure gradient becomes more moderate and wiping performance deteriorates.

As described above, in gas wiping with multi-slot nozzles, the center slot diffusion behavior and the width of the mixed jet change remarkably depending on the flow of the upper/lower auxiliary slot jets, and wiping performance is affected by these changes. Thus, in addition to estimation of the impinging gas pressure, this study revealed that the turbulence in the flow field is also an important parameter for designing a 3-slot nozzle and optimizing its operational conditions.

\section{REFERENCES}

1) Y. Takeishi and T. Aoki: Tetsu-to-Hagané, 81 (1995), 47.

2) J. A. Thornton and H. F. Graff: Metall. Trans. B, 7B (1976), 607

3) C. H. Ellen and C. V. Tu: Trans. ASME, J. Fluid Eng., 106 (1984), 399.

4) Y. Takeishi, A. Yamauchi and S. Miyauchi: Tetsu-to-Hagané, 81 (1995), 37.

5) M. Dubois and J. Callegari: 8th Int. Conf. on Zinc and Zinc Alloy Coated Sheet Steels, Galvatech'11, AIM, Milano, (2011), 845.

6) Y. Takeishi, Y. Suzuki and K. Yabuki: Jpn. J. Multiph. Flow, 14 (2000), No. 2, 194

7) P. Tamadonfar, J. R. McDermid, A. N. Hrymak and F. E. Goodwin: 8th Int. Conf. on Zinc and Zinc Alloy Coated Sheet Steels, Galvatech'11, AIM, Milano, (2011), 817.

8) M. Dubois: 8th Int. Conf. on Zinc and Zinc Alloy Coated Sheet Steels, Galvatech'11, AIM, Milano, (2011), 787.

9) G. Takeda, H. Takahashi, M. Miyake and N. Nakata: Jpn. J. Multiph. Flow, 28 (2014), No. 1, 90.

10) G. Takeda, H. Takahashi, M. Miyake and N. Nakata: CAMP-ISIJ, 26 (2014), 816, CD-ROM.

11) G. Takeda, H. Takahashi, M. Miyake, K. Kabeya and N. Nakata: Asia Steel Int. Conf. 2015 Proc., ISIJ, Tokyo, (2015), 124.

12) T. Shakouchi and T. Kato: Trans. Jpn. Soc. Mech. Eng., 63-614B (1997), 3278.

13) H. Takahashi, G. Takeda, M. Miyake and N. Nakata: J. Jpn. Soc. Exp. Mech., 15 (2015), No. 3, 217.

14) T.-H. Shih, W. W. Liou, A. Shabbir and J. Zhu: Comput. Fluids, 24 (1995), No. 3, 227

15) B. Kader: Int. J. Heat Mass Transf., 24 (1981), No. 9, 1541. 